\title{
Socioeconomic Status and Current Cigarette Smoking Status: Immigrants' Diminished Returns
}

\author{
Shervin Assari ${ }^{*}$ \\ ${ }^{1}$ Department of Family Medicine, Charles R. Drew University of Medicine and Science, Los Angeles, USA
}

Corresponding Author: Shervin Assari, MD, MPH, Assistant Professor, Department of Family Medicine, Charles R. Drew University of Medicine and Science, Los Angeles, CA, USA. Tel: +1-734-2320445, Fax: +1-734-615-8739,

Email: assari@umich.edu

Received October 5, 2019; Accepted April 15, 2020; Online Published April 30, 2020

\begin{abstract}
Introduction: Although socioeconomic status (SES) resources influence population and individual health behaviors, socially marginalized groups gain significantly less health from their SES indicators, such as education and income, compared to the socially privileged groups. This pattern is called marginalization-related diminished returns (MDRs). However, most of the MDRs literature is derived from studies that have defined marginalization based on race and ethnicity. As a result, more research is needed on MDRs due to immigration. To extend what is known about MDRs due to immigration, the current study compared a national sample of immigrants and non-immigrants for the effects of education and income on current cigarette smoking of adults in the United States.

Methods: This is a cross-sectional study. The 2015 National Health Interview Survey (NHIS) enrolled 14,149 individuals who were either immigrants $(n=1977 ; 14.0 \%)$ or non-immigrants $(n=12,166 ; 86.0 \%)$. The independent variables (IV) were education and income that were treated as categorical variables. The dependent variable was current cigarette smoking. Age, gender, race, ethnicity, marital status, employment, and region were confounders. Immigration was the moderator. Logistic regression was used for data analysis.

Results: High education and income were associated with lower odds of current cigarette smoking. However, immigration showed significant statistical interactions with both education and income. These interactions were suggestive of smaller protective effects of high education and income on current cigarette smoking for immigrant than non-immigrant adults.

Conclusion: In line with the MDRs, the effects of education and income on tobacco use is weaker for immigrant than non-immigrant adults.

Keywords: Ethnic Groups, Immigration, Immigrants, Socioeconomic Factors, Tobacco Use, Smoking
\end{abstract}

Citation: Assari S. Socioeconomic status and current cigarette smoking status: immigrants' diminished returns. Int J Travel Med Glob Health 2020;8(2):66-72. doi:10.34172/ijtmgh.2020.11.

\section{Introduction}

Extensive theoretical and empirical work has established the strong effects of socioeconomic status (SES) indicators such as education and income on health outcomes. ${ }^{1-3}$ High education and income are associated with higher happiness ${ }^{4}$ and lower risk of depression, ${ }^{5}$ anxiety ${ }^{6}$ suicide,${ }^{7}$ distress, ${ }^{8,9}$ and substance use. ${ }^{10,11}$ In contrast, immigration is associated with lower SES, poor health, and substance use. ${ }^{12-15}$

Marginalized and non-marginalized groups, however, show health effects of SES indicators such as education and income unequally. ${ }^{16-22}$ According to the Marginalizationrelated Diminished Returns (MDRs) theory, ${ }^{23,24}$ the effects of education, ${ }^{25}$ income, ${ }^{26-30}$ employment, ${ }^{31,32}$ and marital status on mental health, ${ }^{33,34}$ physical health, ${ }^{35-38}$ and health behaviors ${ }^{25,39-41}$ are weaker for minority groups than the majority group. This pattern has been established for Blacks, ${ }^{17,18}$ Hispanics, ${ }^{26,42}$ Asian Americans, ${ }^{43}$ Native Americans, ${ }^{10}$ and the LGBT community. ${ }^{44-46}$ For example, high SES LGBT individuals remain at high risk of loss of mental well-being, ${ }^{44}$ obesity, ${ }^{46}$ and smoking. ${ }^{45}$

According to the MDRs literature, minority status may reduce the health returns of education, ${ }^{35}$ income, ${ }^{26-30}$ occupation, ${ }^{31,32}$ and marital status ${ }^{47}$; however, it is yet unknown if the same MDRs can also be seen for immigrants. That is, it is unclear if immigrants and non-immigrants similarly gain health from their SES resources such as education and income. Similar to other sources of marginalization, namely race, ${ }^{33}$ ethnicity, ${ }^{26,40}$ and sexual orientation, ${ }^{44-46}$ immigrants are pushed to the margin of the host society. Given the immigration rules and regulations, border control laws, and the xenophobia pervasive in our society, immigrants are commonly discriminated against and are treated as secondclass citizens. ${ }^{48-52}$

In this study, the National Health Interview Survey (NHIS), a nationally representative study, was used to compare immigrants and non-immigrants for the effects of education

Copyright $\odot 2020$ The Author(s). This is an open-access article distributed under the terms of the Creative Commons Attribution License (http:// creativecommons.org/licenses/by/4.0), which permits unrestricted use, distribution, and reproduction in any medium, provided the original work is properly cited. 
and income on the current cigarette smoking status of adults in the United States. As suggested by MDRs, immigration was considered as a social identity and social status that reflects marginalization. Thus, weaker effects of education and income on current cigarette smoking of immigrant than non-immigrant people were expected, similar to the pattern observed for Blacks, Hispanics, Asian Americans, Native Americans, and the LGBT community.

\section{Methods}

This is a cross-sectional study. 2015 NHIS data was used. The NHIS is the primary source of information regarding the physical health status of American adults 18 years or older. The NHIS sample is composed of US residents who are civilian, non-institutionalized people. The current analysis is limited to adults.

\section{Participants and Sampling}

The NHIS used a multi-stage clustered/stratified random sampling: First was to sample 428 primary sampling units (PSUs) drawn from 1900 geographically defined PSUs. All the 50 US states and the District of Columbia had representative PSUs in the sample. The PSUs were either a metropolitan statistical area, a single county, or a small group of contiguous counties. For this analysis, only data from adults was used.

\section{Process}

The data is collected by the National Center for Health Statistics (NCHS), which is a part of the Centers for Disease Control and Prevention (CDC). The US Census Bureau collects the data. Data is collected via face-to-face interviews in participants' households. On some occasions, this face-toface interview is followed or replaced by a telephone interview.

\section{Participants}

The total sample in this study was 14,149 adults who were either immigrants $(n=1977 ; 14.0 \%)$ or non-immigrants $(n=12166 ; 86.0 \%)$. People could be of any race/ethnicity to be included in this study. The study had no exclusion criteria.

\section{Measures}

\section{Predictor}

Education. Education is shown to be in 4 categorical levels in Tables 1 and 2 though.

Income. Annual income was self-reported. This variable was measured as a continuous variable in dollars. For the current analysis, however, a dichotomous variable with a cut-off point of 35000 USD was used. Individuals with high income were coded as one, and those with lower income were coded as 0 .

\section{Moderator}

Immigration status. Nativity was self-reported. All participants were asked if they were born in the US. The responses were coded 1 for immigrants and 0 for non-immigrants.

\section{Covariates}

Demographic factors included age, gender, region, race, ethnicity, education, marital status, and employment. Age (years) was a continuous variable. Gender was a dichotomous measure $($ male $=1$, female $=0)$. Participants indicated their region as being either the Northeast, Midwest, South, or West. Participants self-identified their race and ethnicity, both of which were operationalized as categorical variables. Race was White only (reference category), Black/African American only, Native American/Alaska Native only, Asian only, multiple race, and race group not releasable (masked or missing). Ethnicity was Hispanics $=1$, non-Hispanics $=0$ (reference category). Participants were asked about the number of years of schooling. Marital status was a dichotomous variable with married coded as 1. Employment status was a dichotomous variable with employed coded as 1 .

\section{Dependent Variable}

Current Cigarette Smoking. Current cigarette smoking status was the outcome. Smoking status was self-reported. Current cigarette smokers were defined as those who had smoked at least 100 cigarettes, were a smoker at the time of the survey, and reported smoking daily.

\section{Statistical Analyses}

Given the NHIS's multi-stage sampling design, SPSS 23.0 (IBM Inc., NY, USA) was applied for data analysis. Using SPSS, adjustments were made for the NHIS survey weights due to the design variables (strata, clusters, and non-response). Taylor series linearization was applied for the re-estimation of standard errors (SE). Weighted means and frequencies were used for descriptive statistics. For multivariable analyses, four logistic regression models were applied. In these models, education and income were the independent variables; current cigarette smoking status was the dependent variable; and demographic factors and race, ethnicity, and region were the control variables. Immigration status was the moderator. The first two models were calculated in the pooled sample that included both immigrants and non-immigrants. Model $1 \mathrm{did}$ not include immigration by education and income interaction terms. Model 2, however, included immigration by education and income interaction terms. Model 3 and Model 4 were performed in non-immigrants and immigrants, respectively. Adjusted odds ratio (OR), 95\% confidence intervals (CI), SE, and $P$ values were reported. A $P$ value of less than 0.05 was considered significant.

\section{Results}

\section{Descriptive Statistics}

The total sample in this study was 14149 immigrant and nonimmigrant American adults 55+ years old. Table 3 depicts the descriptive statistics of the participants overall and based on nativity.

\section{Pooled Sample Logistic Regressions}

Table 1 shows the results of two logistic regressions in the pooled sample with education and income as the predictors and current cigarette smoking status as the outcome (dependent variable). Model 1 included only the main effects of education and income; however, Model 2 added the interaction terms between immigration status, education, and 
Table 1. Logistic Regressions in the Pooled Sample ( $\mathrm{n}=14149)$

\begin{tabular}{|c|c|c|c|c|c|c|c|c|}
\hline \multirow[b]{3}{*}{ Immigrant } & \multicolumn{4}{|c|}{ Model 1} & \multicolumn{4}{|c|}{ Model 2} \\
\hline & \multirow{2}{*}{$\frac{\text { OR }}{0.70}$} & \multicolumn{2}{|c|}{$95 \% \mathrm{Cl}$} & \multirow{2}{*}{$\frac{\boldsymbol{P}}{0.000}$} & \multirow{2}{*}{$\frac{\text { OR }}{0.36}$} & \multicolumn{2}{|c|}{$95 \% \mathrm{Cl}$} & \multirow{2}{*}{$\frac{\boldsymbol{P}}{0.000}$} \\
\hline & & 0.60 & 0.81 & & & 0.27 & 0.47 & \\
\hline Ethnicity (Hispanic) & 0.48 & 0.41 & 0.55 & 0.000 & 0.53 & 0.46 & 0.61 & 0.000 \\
\hline Race & & & & 0.000 & & & & 0.000 \\
\hline \multicolumn{9}{|l|}{ White only } \\
\hline Black/African American only & 0.64 & 0.56 & 0.73 & 0.000 & 0.62 & 0.54 & 0.71 & 0.000 \\
\hline AIAN only & 1.01 & 0.71 & 1.45 & 0.941 & 0.99 & 0.69 & 1.41 & 0.942 \\
\hline Asian only & 0.83 & 0.66 & 1.05 & 0.117 & 0.71 & 0.56 & 0.90 & 0.004 \\
\hline Multiple races & 1.03 & 0.79 & 1.34 & 0.847 & 1.01 & 0.78 & 1.32 & 0.916 \\
\hline Race group not releasable & 0.51 & 0.22 & 1.21 & 0.128 & 0.56 & 0.24 & 1.32 & 0.187 \\
\hline Male & 1.27 & 1.17 & 1.38 & 0.000 & 1.26 & 1.16 & 1.38 & 0.000 \\
\hline Age & 0.99 & 0.99 & 1.00 & 0.000 & 0.99 & 0.99 & 1.00 & 0.000 \\
\hline \multicolumn{9}{|l|}{ Region } \\
\hline \multicolumn{9}{|l|}{ West } \\
\hline Northeast & 0.99 & 0.86 & 1.13 & 0.865 & 0.98 & 0.86 & 1.13 & 0.806 \\
\hline Midwest & 1.17 & 1.04 & 1.31 & 0.010 & 1.16 & 1.03 & 1.31 & 0.013 \\
\hline South & 1.17 & 1.05 & 1.31 & 0.005 & 1.16 & 1.04 & 1.30 & 0.008 \\
\hline Married & 0.60 & 0.55 & 0.66 & 0.000 & 0.61 & 0.56 & 0.67 & 0.000 \\
\hline Employed & 0.95 & 0.85 & 1.06 & 0.372 & 0.96 & 0.86 & 1.08 & 0.495 \\
\hline Education & & & & 0.000 & & & & 0.000 \\
\hline \multicolumn{9}{|l|}{ Less than 12 years } \\
\hline 12 years & 0.81 & 0.70 & 0.94 & 0.005 & 0.70 & 0.60 & 0.83 & 0.000 \\
\hline 13-15 years & 0.51 & 0.44 & 0.60 & 0.000 & 0.43 & 0.36 & 0.51 & 0.000 \\
\hline $16+$ years & 0.23 & 0.20 & 0.27 & 0.000 & 0.18 & 0.15 & 0.22 & 0.000 \\
\hline Income $\geq 35000$ USD & 0.69 & 0.63 & 0.76 & 0.000 & 0.64 & 0.58 & 0.71 & 0.000 \\
\hline Education $\mathrm{x}$ Immigrant & & & & & & & & 0.000 \\
\hline 12 Years x Immigrant & & & & & 1.30 & 0.91 & 1.86 & 0.149 \\
\hline $13-15$ years $x$ Immigrant & & & & & 1.94 & 1.34 & 2.81 & 0.000 \\
\hline $16+$ Years $x$ Immigrant & & & & & 2.63 & 1.81 & 3.83 & 0.000 \\
\hline Income $\geq 35000$ USD x Immigrant & & & & & 1.72 & 1.32 & 2.25 & 0.000 \\
\hline Constant & 0.82 & & & .084 & 0.97 & & & 0.797 \\
\hline
\end{tabular}

income. Based on Model 1, high education and income were linked to lower odds of current cigarette smoking. Model 2, however, revealed statistically significant interactions between education and income with immigration status on current cigarette smoking status of adults. The model suggested that the protective effects of education and income against current cigarette smoking status were smaller for immigrant than non-immigrant adults (Table 1).

\section{Stratified Logistic Regressions}

Table 2 shows the results of one logistic regression on non-immigrants (Model 3) and one logistic regression in immigrants (Model 4). In these models, education and income were the predictors, and current cigarette smoking status was the outcome (dependent variable). Based on Model 3, high education levels and income were associated with lower odds of current cigarette smoking for non-immigrant adults. Model 4 did not show protective effects of most education levels and income on current cigarette smoking for immigrant adults (Table 2).

\section{Discussion}

Education and income were associated with lower odds of current cigarette smoking status of adults. These effects, however, were larger for non-immigrants than immigrants.

Marginalization, broadly defined, reduces the health return of education and income among other SES indicators. This is supported by the observations that Blacks, ${ }^{33}$ Hispanics, ${ }^{26,40}$ Asian Americans, ${ }^{43}$ Native Americans, ${ }^{10}$ and people of the LGBT community ${ }^{46}$ show MDRs. If any type of marginalization reduces the health gains that follow SES indicators such as education and income, similar patterns should be expected for immigrants. ${ }^{48-52}$ Societal and structural factors such as social stratification, residential segregation, labor market discrimination, and low availability of resources in urban areas may all suggest that immigrants may be less likely to benefit from their education and income fully.

As education and income improve, tobacco use reduces, but less for immigrants than non-immigrants. This finding is an extension of previous literature on $\mathrm{MDRs}^{23,24}$ on diminishing returns of education, ${ }^{35}$ income, ${ }^{26-30}$ occupation, ${ }^{31,32}$ and marital status ${ }^{47}$ on a wide range of mental health outcomes 
Table 2. Logistic Regressions in Non-immigrants and Immigrants $(n=14149)$

\begin{tabular}{|c|c|c|c|c|c|c|c|c|}
\hline \multirow[b]{3}{*}{ Ethnicity (Hispanic) } & \multicolumn{4}{|c|}{ Model 3} & \multicolumn{4}{|c|}{ Model 4} \\
\hline & \multirow{2}{*}{$\begin{array}{c}\text { OR } \\
0.52\end{array}$} & \multicolumn{2}{|c|}{$95 \% \mathrm{Cl}$} & \multirow{2}{*}{$\begin{array}{c}\boldsymbol{P} \\
<0.001\end{array}$} & \multirow{2}{*}{$\begin{array}{c}\text { OR } \\
0.53\end{array}$} & \multicolumn{2}{|c|}{$95 \% \mathrm{Cl}$} & \multirow{2}{*}{$\begin{array}{c}\frac{\boldsymbol{P}}{<0.001} \\
<\end{array}$} \\
\hline & & 0.44 & 0.62 & & & 0.38 & 0.74 & \\
\hline \multicolumn{9}{|l|}{ White only } \\
\hline Black/African American only & 0.64 & 0.55 & 0.73 & $<0.001$ & 0.48 & 0.31 & 0.74 & 0.001 \\
\hline AIAN only & 1.05 & 0.72 & 1.53 & 0.819 & 0.60 & 0.18 & 2.01 & 0.407 \\
\hline Asian only & 0.61 & 0.40 & 0.92 & 0.020 & 0.73 & 0.52 & 1.02 & 0.065 \\
\hline Multiple races & 1.04 & 0.78 & 1.37 & 0.791 & 0.86 & 0.37 & 1.98 & 0.723 \\
\hline Race group not releasable & 0.66 & 0.22 & 1.93 & 0.443 & 0.42 & 0.10 & 1.82 & 0.246 \\
\hline Male & 1.13 & 1.03 & 1.24 & 0.008 & 2.77 & 2.16 & 3.56 & $<0.001$ \\
\hline Age & 0.99 & 0.99 & 1.00 & $<0.001$ & 1.00 & 0.99 & 1.01 & 0.474 \\
\hline \multicolumn{9}{|l|}{ Region } \\
\hline Northeast & 0.98 & 0.84 & 1.14 & 0.780 & 1.04 & 0.75 & 1.45 & 0.816 \\
\hline Midwest & 1.21 & 1.07 & 1.37 & 0.003 & 0.79 & 0.54 & 1.15 & 0.218 \\
\hline South & 1.19 & 1.06 & 1.35 & 0.004 & 0.97 & 0.74 & 1.27 & 0.811 \\
\hline Married & 0.63 & 0.57 & 0.69 & $<0.001$ & 0.49 & 0.38 & 0.61 & $<0.001$ \\
\hline Employed & 0.96 & 0.85 & 1.08 & 0.519 & 0.92 & 0.67 & 1.28 & 0.637 \\
\hline Education & & & & $<0.001$ & & & & 0.001 \\
\hline \multicolumn{9}{|l|}{ Less than 12 years } \\
\hline 12 years & 0.70 & 0.59 & 0.83 & $<0.001$ & 0.96 & 0.69 & 1.34 & 0.822 \\
\hline 13-15 years & 0.42 & 0.36 & 0.50 & $<0.001$ & 0.95 & 0.66 & 1.36 & 0.768 \\
\hline $16+$ Years & 0.18 & 0.15 & 0.21 & $<0.001$ & 0.54 & 0.37 & 0.78 & 0.001 \\
\hline Income $\geq 35000$ USD & 0.66 & 0.60 & 0.73 & $<0.001$ & 0.95 & 0.73 & 1.23 & 0.684 \\
\hline Constant & 1.05 & & & 0.694 & 0.18 & & & $<0.001$ \\
\hline
\end{tabular}

such as psychological distress, ${ }^{53}$ depression, ${ }^{27,54}$ suicide,${ }^{33}$ and anxiety. ${ }^{47}$ Similar MDRs are reported for smoking, ${ }^{10,41,45,55,56}$ vaping, ${ }^{39}$ drinking, ${ }^{40,57}$ diet, ${ }^{58}$ and exercise, ${ }^{13}$ Finally, MDRs are seen for physical health outcomes such as obesity, ${ }^{35,36}$ self-rated health, ${ }^{26,34,53}$ chronic diseases, ${ }^{28,29,37}$ disability, ${ }^{59}$ and mortality. ${ }^{38}$ Not only educational attainment ${ }^{34}$ and income, ${ }^{28}$ but also occupation, ${ }^{38}$ and marital status ${ }^{47}$ all generate less health for the majority than marginalized people. Social marginalization, regardless of its type, whether it is based on race, ${ }^{60}$ ethnicity, ${ }^{26,40}$ sexual orientation, ${ }^{44-46}$ or immigration are consistently associated with less health gain from income and other SES indicators. ${ }^{60}$

The robust and consistent nature of the MDRs shifts the blame from the marginalized people to the society that reduces the gain of all vulnerable and disadvantaged groups. Thus, these patterns suggest that the MDRs are due to the function and structure of society. US social institutions treat people differentially based on their color, race, ethnicity, class, heritage, and nativity, resulting in systemic marginalization of non-majority groups. Such marginalization reduces people's chances for enjoying full participation and full benefits from resources that are available to them. The racism, xenophobia, and nationalism embedded in the social fabric of the US society reduce immigrants, LGBTs, and racial and ethnic minorities' ability to fully leverage their human capital and turn it into tangible outcomes. As a result, they show less than expected benefits in the presence of education, income, and other SES resources. ${ }^{23,24}$

\section{Implications}

To undo MDRs, bold policies are needed that can equalize the health return of education and income as well as other SES indicator cross groups. Such policies should go beyond equal access to education and income and focus on equality in the returns of SES indicators across social groups. Specific policies and programs should help immigrants to more effectively mobilize and leverage their education and income to gain tangible outcomes. Ways by which the purchasing power of immigrants can be enhanced should be studied in future research.

\section{Limitations}

The current results should be interpreted with the methodological limitations in mind. First, any cross-sectional study is limited in drawing causal inferences. It cannot be ruled out that excessive health problems influence social mobility and the ability to generate educational mobility and income. Thus, reverse causality cannot be ruled out in this study. Thus, the results should be interpreted not as causation, but as association. Moreover the mechanisms by which MDRs of education and income emerge were not studied. The lower purchasing power of income for immigrants may be the mechanism. Access to the country of origin was not available; nor did we control for type of occupation, wealth, assets, 
Table 3. Descriptive Statistics Overall and Based on Immigration ( $n=14149)$

\begin{tabular}{|c|c|c|c|c|c|c|}
\hline & \multicolumn{2}{|c|}{ All } & \multicolumn{2}{|c|}{ Non-Immigrant } & \multicolumn{2}{|c|}{ Immigrant } \\
\hline & No. & $\%$ & No. & $\%$ & No. & $\%$ \\
\hline \multicolumn{7}{|l|}{ Immigrants } \\
\hline No & 12166 & 86.0 & 12166 & 100.0 & - & - \\
\hline Yes & 1977 & 14.0 & - & - & 1977 & 100.0 \\
\hline \multicolumn{7}{|l|}{ Ethnicity* } \\
\hline Non-Hispanic & 12698 & 89.7 & 11668 & 95.9 & 1026 & 51.9 \\
\hline \multicolumn{7}{|l|}{ Race* } \\
\hline White only & 11351 & 80.2 & 10047 & 82.6 & 1298 & 65.7 \\
\hline Black/African American only & 1843 & 13.0 & 1671 & 13.7 & 172 & 8.7 \\
\hline AIAN only & 123 & 0.9 & 98 & 0.8 & 25 & 1.3 \\
\hline Asian only & 599 & 4.2 & 148 & 1.2 & 451 & 22.8 \\
\hline Multiple races & 218 & 1.5 & 194 & 1.6 & 24 & 1.2 \\
\hline Race group not releasable & 15 & 0.1 & 8 & 0.1 & 7 & 0.4 \\
\hline Women & 8079 & 57.1 & 6917 & 56.9 & 1159 & 58.6 \\
\hline Men & 6070 & 42.9 & 5249 & 43.1 & 818 & 41.4 \\
\hline \multicolumn{7}{|l|}{ Region* } \\
\hline Northeast & 2531 & 17.9 & 2030 & 16.7 & 501 & 25.3 \\
\hline Midwest & 3002 & 21.2 & 2836 & 23.3 & 166 & 8.4 \\
\hline South & 4881 & 34.5 & 4262 & 35.0 & 617 & 31.2 \\
\hline West & 3735 & 26.4 & 3038 & 25.0 & 693 & 35.1 \\
\hline \multicolumn{7}{|l|}{ Marital status* } \\
\hline Non-Married & 7769 & 54.9 & 6777 & 55.7 & 990 & 50.1 \\
\hline Married & 6380 & 45.1 & 5389 & 44.3 & 987 & 49.9 \\
\hline \multicolumn{7}{|l|}{ Employed } \\
\hline No & 9759 & 69.0 & 8429 & 69.3 & 1326 & 67.1 \\
\hline Yes & 4390 & 31.0 & 3737 & 30.7 & 651 & 32.9 \\
\hline 12 years & 3898 & 27.7 & 3476 & 28.7 & 421 & 21.6 \\
\hline $13-15$ years & 3519 & 25.0 & 3210 & 26.5 & 309 & 15.9 \\
\hline $16+$ years & 4388 & 31.2 & 3834 & 31.7 & 550 & 28.3 \\
\hline \multicolumn{7}{|l|}{ Income $>35 \mathrm{k}^{*}$} \\
\hline No & 2137 & 47.9 & 1790 & 46.8 & 347 & 54.8 \\
\hline Yes & 2324 & 52.1 & 2038 & 53.2 & 286 & 45.2 \\
\hline \multicolumn{7}{|l|}{ Current smoking* } \\
\hline No & 28126 & 83.9 & 22480 & 82.3 & 5631 & 90.7 \\
\hline \multirow[t]{2}{*}{ Yes } & 5415 & 16.1 & 4836 & 17.7 & 576 & 9.3 \\
\hline & Mean & SD & Mean & SD & Mean & SD \\
\hline Age* & 68.17 & 9.09 & 68.28 & 9.13 & 67.55 & 8.80 \\
\hline
\end{tabular}

${ }^{*} P<0.05$ for comparison of immigrants and non-immigrants.

or parental education. Future research should replicate and validate these findings using longitudinal data with a more comprehensive list of measures on nativity, country of origin, and other SES indicators. Future research may also include contextual factors such as neighborhoods' ethnic composition, SES, or density of resources as factors that may cause MDRs. It is likely that highly educated and high-income immigrants report poor mental health, because they need to spend more time on the job or they face extra stress to gain such education and income. Finally, there is a need to compare immigrants from Asia, Africa, and Latino countries, as each culture may adopt US culture differently.

\section{Conclusion}

While education and income reduce the odds of current cigarette smoking status of American adults, these influences are weaker for immigrants than non-immigrants. Thus, tobacco use disparities in immigrants are beyond SES inequalities and also diminishing marginal returns of SES indicators such as education and income for immigrants. To eliminate health inequalities that impact immigrants, it is essential to recognize and address MDRs-related inequalities that endure across all SES levels.

\section{Conflict of Interest Disclosures}

The author declares no conflicts of interest.

\section{Ethical Approval}

All participants signed written consent forms. The NHIS protocol was approved by the CDC Instructional Review 


\section{Research Highlights}

\section{What Is Already Known?}

High education and income are associated with lower odds of current cigarette smoking; however, these effects may vary across population sub-groups.

\section{What This Study Adds?}

In line with the marginalization-related diminished returns, education and income generate less protection against cigarette smoking for immigrants than non-immigrants in the United States. As a result, we may observe a higher than expected prevalence of smokers among highly educated and high-income immigrants.

Board (IRB). According to the NIH guideline as well as the decision tool regarding human subject research, secondary analyses of publicly available fully de-identified existing data are "Non-Human Subject Research." The definition of the "Non-Human Subject Research" as well as the decision tool are available here: https:/grants.nih.gov/policy/humansubjects/ hs-decision.htm. Non-human subject research is exempt from the IRB review.

\section{Funding/Support}

SA is supported by the NIH under Awards 54MD008149, R25 MD007610, 2U54MD007598, and U54 TR001627. SA is also supported by D084526-03 and CA201415-02 as well as the Center for Medicare and Medicaid Services (CMS) Grant 1H0CMS331621.

\section{References}

1. Marmot M. Social determinants of health inequalities. Lancet. 2005;365(9464):1099-1104. doi:10.1016/s0140-6736(05)711466.

2. Marmot M. The Status Syndrome: How Social Standing Affects Our Health and Longevity. London: Bloomsbury Press; 2004.

3. Marmot M. Economic and social determinants of disease. Bull World Health Organ. 2001;79(10):988-989.

4. Assari S. Race, education attainment, and happiness in the United States. Int J Epidemiol Res. 2019;6(2):76-82. doi:10.15171/ ijer.2019.14

5. Hudson DL, Puterman E, Bibbins-Domingo K, Matthews KA, Adler NE. Race, life course socioeconomic position, racial discrimination, depressive symptoms and self-rated health. Soc Sci Med. 2013;97:7-14. doi:10.1016/j.socscimed.2013.07.031.

6. Murcia M, Chastang JF, Niedhammer I. Educational inequalities in major depressive and generalized anxiety disorders: results from the French national SIP study. Soc Psychiatry Psychiatr Epidemiol. 2015;50(6):919-928. doi:10.1007/s00127-015-1010-9.

7. Phillips JA, Hempstead K. Differences in US suicide rates by educational attainment, 2000-2014. Am J Prev Med. 2017;53(4):e123-e130. doi:10.1016/j.amepre.2017.04.010.

8. Merz EC, Tottenham N, Noble KG. Socioeconomic status, amygdala volume, and internalizing symptoms in children and adolescents. J Clin Child Adolesc Psychol. 2018;47(2):312-323. doi:10.1080/15374416.2017.1326122.

9. Ursache A, Merz EC, Melvin S, Meyer J, Noble KG. Socioeconomic status, hair cortisol and internalizing symptoms in parents and children. Psychoneuroendocrinology. 2017;78:142-150. doi:10.1016/j.psyneuen.2017.01.020.
10. Assari S, Bazargan M. Protective effects of educational attainment against cigarette smoking; diminished returns of American Indians and Alaska natives in the National Health Interview Survey. Int J Travel Med Glob Health. 2019;7(3):105-110. doi:10.15171/ ijtmgh.2019.22.

11. Ergin I, Hassoy H, Tanik FA, Aslan G. Maternal age, education level and migration: socioeconomic determinants for smoking during pregnancy in a field study from Turkey. BMC Public Health. 2010;10:325. doi:10.1186/1471-2458-10-325.

12. Assari S. Socioeconomic determinants of systolic blood pressure; minorities' diminished returns. J Health Econ Dev. 2019;1(1):1-11.

13. Assari S. Educational attainment and exercise frequency in American women; blacks' diminished returns. Womens Health Bull. 2019;6(3). doi:10.5812/whb.87413.

14. Assari S. Ethnicity, educational attainment, and physical health of older adults in the United States. Aging Med (Milton). 2019;2(2):104-111. doi:10.1002/agm2.12050.

15. Assari S. Parental educational attainment and academic performance of American college students; blacks' diminished returns. J Health Econ Dev. 2019;1(1):21-31.

16. Assari S. Cross-country differences in the additive effects of socioeconomics, health behaviors and medical comorbidities on disability among older adults with heart disease. J Tehran Heart Cent. 2015;10(1):24-33.

17. Assari S, Moghani Lankarani M. Does multi-morbidity mediate the effect of socioeconomics on self-rated health? Cross-country differences. Int J Prev Med. 2015;6:85. doi:10.4103/20087802.164413 .

18. Assari S. Cross-country variation in additive effects of socioeconomics, health behaviors, and comorbidities on subjective health of patients with diabetes. J Diabetes Metab Disord. 2014;13(1):36. doi:10.1186/2251-6581-13-36.

19. Assari S, Moghani Lankarani R, Moghani Lankarani M. Crosscountry differences in the association between diabetes and disability. J Diabetes Metab Disord. 2014;13(1):3. doi:10.1186/2251-6581-13-3

20. Hudson DL, Bullard KM, Neighbors HW, Geronimus AT, Yang J, Jackson JS. Are benefits conferred with greater socioeconomic position undermined by racial discrimination among African American men? J Mens Health. 2012;9(2):127-136. doi:10.1016/j. jomh.2012.03.006.

21. Hudson DL, Neighbors HW, Geronimus AT, Jackson JS. The relationship between socioeconomic position and depression among a US nationally representative sample of African Americans. Soc Psychiatry Psychiatr Epidemiol. 2012;47(3):373381. doi:10.1007/s00127-011-0348-x.

22. Hudson DL, Neighbors HW, Geronimus AT, Jackson JS. Racial discrimination, John Henryism, and depression among African Americans. J Black Psychol. 2016;42(3):221-243. doi:10.1177/0095798414567757.

23. Assari S. Health disparities due to diminished return among black Americans: public policy solutions. Social Issues and Policy Review. 2018;12(1):112-145. doi:10.1111/sipr.12042.

24. Assari S. Unequal gain of equal resources across racial groups. Int J Health Policy Manag. 2018;7(1):1-9. doi:10.15171/ ijhpm.2017.90.

25. Assari S, Mistry R. Educational attainment and smoking status in a national sample of American adults; evidence for the blacks' diminished return. Int J Environ Res Public Health. 2018;15(4). doi:10.3390/ijerph15040763.

26. Assari S. Socioeconomic status and self-rated oral health; diminished return among Hispanic whites. Dent J (Basel). 2018;6(2). doi:10.3390/dj6020011.

27. Assari S. High income protects whites but not African Americans against risk of depression. Healthcare (Basel). 2018;6(2). doi:10.3390/healthcare6020037.

28. Assari S. The benefits of higher income in protecting against chronic medical conditions are smaller for African Americans than whites. 
Healthcare(Basel). 2018;6(1). doi:10.3390/healthcare6010002.

29. Assari S, Caldwell $\mathrm{CH}$. Family income at birth and risk of attention deficit hyperactivity disorder at age 15: racial differences. Children (Basel). 2019;6(1). doi:10.3390/children6010010.

30. Assari S, Hani N. Household income and children's unmet dental care need; blacks' diminished return. Dent J (Basel). 2018;6(2). doi:10.3390/dj6020017.

31. Assari S. Life Expectancy gain due to employment status depends on race, gender, education, and their intersections. J Racial Ethn Health Disparities. 2018;5(2):375-386. doi:10.1007/s40615-0170381-x.

32. Assari S, Bazargan M. Unequal associations between educational attainment and occupational stress across racial and ethnic groups. Int J Environ Res Public Health. 2019;16(19). doi:10.3390/ ijerph16193539.

33. Assari S, Schatten HT, Arias SA, Miller IW, Camargo CA, Boudreaux ED. Higher educational attainment is associated with lower risk of a future suicide attempt among non-Hispanic whites but not nonHispanic blacks. J Racial Ethn Health Disparities. 2019;6(5):10011010. doi:10.1007/s40615-019-00601-z.

34. Assari S. Blacks' Diminished return of education attainment on subjective health; mediating effect of income. Brain Sci. 2018;8(9). doi:10.3390/brainsci8090176

35. Assari S. Family income reduces risk of obesity for white but not black children. Children (Basel). 2018;5(6). doi:10.3390/ children5060073

36. Assari S, Thomas A, Caldwell CH, Mincy RB. Blacks' diminished health return of family structure and socioeconomic status; 15 years of follow-up of a national urban sample of youth. J Urban Health. 2018;95(1):21-35. doi:10.1007/s11524-017-0217-3.

37. Assari S, Moghani Lankarani M. Poverty status and childhood asthma in white and black families: National Survey of Children's Health. Healthcare (Basel). 2018;6(2). doi:10.3390/ healthcare6020062.

38. Assari S, Moghani Lankarani M. Race and urbanity alter the protective effect of education but not income on mortality. Front Public Health. 2016;4:100. doi:10.3389/fpubh.2016.00100.

39. Assari S, Mistry R, Bazargan M. Race, educational attainment, and e-cigarette use. J Med Res Innov. 2020;4(1). doi:10.32892/ jmri.185.

40. Assari S, Farokhnia M, Mistry R. Education attainment and alcohol binge drinking: diminished returns of Hispanics in Los Angeles. Behav Sci (Basel). 2019;9(1). doi:10.3390/bs9010009.

41. Assari S, Mistry R. Diminished return of employment on ever smoking among Hispanic whites in Los Angeles. Health Equity. 2019;3(1):138-144. doi:10.1089/heq.2018.0070.

42. Assari S, Bazargan M. Educational attainment and self-rated oral health among American older adults: Hispanics' diminished returns. Dent J (Basel). 2019;7(4). doi:10.3390/dj7040097.

43. Assari S, Boyce S, Bazargan $\mathrm{M}$, Caldwell $\mathrm{CH}$. Mathematical performance of American youth: diminished returns of educational attainment of Asian-American parents. Educ Sci (Basel). 2020;10(2). doi:10.3390/educsci10020032.

44. Assari S, Bazargan M. Educational attainment and subjective health and well-being; diminished returns of lesbian, gay, and bisexual individuals. Behav Sci (Basel). 2019;9(9). doi:10.3390/ bs9090090.
45. Assari S, Bazargan M. Education level and cigarette smoking: diminished returns of lesbian, gay and bisexual individuals. Behav Sci (Basel). 2019;9(10). doi:10.3390/bs9100103.

46. Assari S. Education attainment and obesity: differential returns based on sexual orientation. Behav Sci (Basel). 2019;9(2). doi:10.3390/bs9020016.

47. Assari S, Caldwell $\mathrm{CH}$, Zimmerman MA. Family structure and subsequent anxiety symptoms; minorities' diminished return. Brain Sci. 2018;8(6). doi:10.3390/brainsci8060097.

48. Cobb CL, Meca A, Branscombe NR, et al. Perceived discrimination and well-being among unauthorized Hispanic immigrants: the moderating role of ethnic/racial group identity centrality. Cultur Divers Ethnic Minor Psychol. 2019;25(2):280-287. doi:10.1037/ cdp0000227.

49. Fischer S, Nater UM, Strahler J, et al. Psychobiological impact of ethnic discrimination in Turkish immigrants living in Germany. Stress. 2017;20(2):167-174. doi:10.1080/10253890.2017.12964 30.

50. Flippen CA, Parrado EA. Perceived discrimination among Latino immigrants in new destinations: the case of Durham, NC. Sociol Perspect. 2015;58(4):666-685. doi:10.1177/0731121415574397.

51. Straiton ML, Aambø AK, Johansen R. Perceived discrimination, health and mental health among immigrants in Norway: the role of moderating factors. BMC Public Health. 2019;19(1):325. doi:10.1186/s12889-019-6649-9

52. Yoo HC, Gee GC, Takeuchi D. Discrimination and health among Asian American immigrants: disentangling racial from language discrimination. Soc Sci Med. 2009;68(4):726-732. doi:10.1016/j. socscimed.2008.11.013.

53. Assari S, Lapeyrouse LM, Neighbors HW. Income and selfrated mental health: diminished returns for high income black Americans. Behav Sci (Basel). 2018;8(5). doi:10.3390/bs8050050.

54. Assari S. Educational attainment better protects African American women than African American men against depressive symptoms and psychological distress. Brain Sci. 2018;8(10). doi:10.3390/ brainsci8100182.

55. Assari S, Bazargan M. Unequal effects of educational attainment on workplace exposure to second-hand smoke by race and ethnicity; minorities' diminished returns in the National Health Interview Survey (NHIS). J Med Res Innov. 2019;3(2). doi:10.32892/jmri.179.

56. Assari S, Bazargan M. Second-hand smoke exposure at home in the United States; minorities' diminished returns. Int J Travel Med Glob Health. 2019;7(4):135-141. doi:10.15171/ijtmgh.2019.28.

57. Assari S, Moghani Lankarani M. Education and alcohol consumption among older Americans; black-white differences. Front Public Health. 2016;4:67. doi:10.3389/fpubh.2016.00067.

58. Assari S, Moghani Lankarani M. Educational attainment promotes fruit and vegetable intake for whites but not blacks. J (Basel). 2018;1(1):29-41. doi:10.3390/j1010005.

59. Assari S, Bazargan M. Educational attainment better reduces disability for non-Hispanic than Hispanic Americans. Eur J Investig Health Psychol Educ. 2020;10(1):10-17. doi:10.3390/ ejihpe10010002.

60. Assari S. Parental educational attainment and mental well-being of college students; diminished returns of blacks. Brain Sci. 2018;8(11). doi:10.3390/brainsci8110193. 